\section{Divulgación de información financiera en entidades del sector solidario por medio de herramientas de tecnologías de la información y la comunicación}

\author{
LEIDY KATERINE ROJAS-MOLINA ${ }^{a}$
}

\begin{abstract}
RESUMEN El uso de herramientas de tecnología de la información y la comunicación (TIC) facilita la interacción entre los usuarios y las organizaciones. En entidades del sector público se emplean estos mecanismos como difusores de la información financiera, ya que mejoran los niveles de transparencia puesto que son de fácil acceso y llegan a gran parte de la población. Sin embargo, esta situación se presenta con menos frecuencia en las entidades del sector privado, en las que no suelen divulgar la información de sus estados financieros por medio de herramientas TIC. Existen entidades privadas que son de interés de una gran parte de la población, puesto que constituyen una fuerza económica importante para el país. Tal es el caso de las entidades del sector solidario, por tanto, al considerar el grado de interés del público en dichas entidades, se analiza el porcentaje con que se divulga información relacionada con los estados financieros de organizaciones del sector solidario por medio de herramientas TIC, como, por ejemplo, a través de Internet. Esta medición se realiza con el empleo de un índice de puntuación dicotómica que permite evaluar condiciones relacionadas con la correcta divulgación de información financiera y el grado de cumplimiento de dichas entidades.
\end{abstract}

PALABRAS CLAVE divulgación, estados financieros, tIC.
HISTORIA DEL ARTÍCULO

¿CÓMO CITAR?:

Rojas Molina, L.K. (2018).

Divulgación de información

financiera en entidades del sector solidario por medio de herramientas de tecnologías de la información y la comunicación. Perspectiva Empresarial, 5(1), 79-90. http:// dx.doi.org/10.16967/rpe.v5n1a7

RECIBIDO: 16 de enero de 2018 APROBADO: 17 de febrero de 2018

CORRESPONDENCIA: Leidy Katerine Rojas-Molina, Transversal 17 No. 25-25, Bogotá, Colombia.

a Magíster, Docente de investigación en Fundación San Mateo, Colombia. Correo electrónico: Ikrojas@sanmateo.edu.co 
¿CÓMO CITO EL ARTÍCULO? HOW TO CITE THIS PAPER?

CHICAGO:

Rojas-Molina, Leidy Katerine. 2018. "Divulgación de información financiera en entidades del sector solidario por medio de herramientas de tecnologías de la información y la comunicación". Perspectiva Empresarial 5(1): 79-90. http:// dx.doi.org/10.16967/rpe. v5n1a7

MLA:

Rojas-Molina, Leidy Katerine. "Divulgación de información financiera en entidades del sector solidario por medio de herramientas de tecnologías de la información y la comunicación". Perspectiva Empresarial 5.1 (2018): 7990. Digital. http://dx.doi. org/10.16967/rpe.v5n1a7

\section{Financial Reporting in Entities of the Solidarity Sector through Information and Communication Technology Tools}

ABSTRACT The use of information and communication technology (ICT) tools facilitates the interaction between users and organizations. In public sector entities, these mechanisms are used as transmitters of financial information since they improve levels of transparency, are easily accessible and reach a large part of the population. However, this occurs less frequently in private sector entities, which do not usually disclose the information of their financial statements through ICT tools. There are private entities that are of interest to a large part of the population because they constitute an important economic force for the country. This is the case of entities in the solidarity sector; therefore, in considering the degree of public interest in said entities, we analyze the percentage of information related to the financial statements of organizations in the solidarity sector that is disclosed by means of ICT tools such as the Internet. This is measured using a dichotomous scoring index that allows to assess conditions related to correct financial reporting and said entities' degree of compliance.

KEYWORDS disclosure, financial statements, ICT.

\section{Divulgação de informação financeira em entidades do setor solidário por meio de ferramentas de tecnologias da informação e comunicação}

RESUMO O uso de ferramentas de tecnologia da informação e comunicação (TIC) facilita a interação entre os usuários e as organizações. Em entidades do setor público esses mecanismos são empregados como difusores da informação financeira, já que melhoram os níveis de transparência, posto que são de fácil acesso e chegam a grande parte da população. Porém, essa situação aparece com menos frequência nas entidades do setor privado, nas quais não costumam divulgar a informação dos seus estados financeiros por meio de ferramentas TIC. Existem entidades privadas que são de interesse de uma grande parte da população, posto que constituem uma força econômica importante para o país. Como é o caso das entidades do setor solidário, portanto, ao considerar o nível de interesse do público em ditas entidades, analisa-se a porcentagem com a qual informação relacionada com os estados financeiros de organizações do setor solidário é divulgada por meio de ferramentas TIC, como, por exemplo, através da Internet. Essa medição é realizada com o emprego de um índice de pontuação dicotômica que permite avaliar as condições relacionadas com a correta divulgação da informação financeira e o nível de cumprimento de ditas entidades.

PALAVRAS CHAVE divulgação, estados financeiros, TIC. 


\section{Introducción}

Divulgar información financiera resulta de vital importancia para las organizaciones, ya que una adecuada toma de decisiones depende, en gran medida, de la información financiera que tengan disponible los usuarios. Para esto, las entidades pueden apoyarse en diversos mecanismos que favorecen la comunicación entre los usuarios, mecanismos que se han visto fortalecidos con la implementación de las tecnologías de la información y la comunicación (TIC).

Las TIC abarcan distintos componentes que favorecen la interacción entre los grupos de interés. El uso de Internet es una de las herramientas que más ha favorecido la comunicación en los últimos años, por ser de fácil acceso y permitir una interacción sencilla y oportuna entre todos los usuarios de la información. De esta forma, las entidades del sector público la consideran hoy una herramienta fundamental en la divulgación de la información financiera (Peña, Vargas \& Medina, 2012).

Sin embargo, una situación diferente se presenta con las entidades del sector privado, ya que al no existir una legislación que las obligue a publicar los estados financieros en sus páginas web, la información no tiende a revelarse por estos medios. Por esta razón, en esta investigación se busca identificar el grado de divulgación de los estados financieros con el empleo de tecnologías de la información y la comunicación en entidades vigiladas por la Superintendencia Solidaria, y se analiza, además, si la publicación de la información cumple con aspectos mínimos que permitan a los usuarios comprender los hechos económicos.

Algunos autores han analizado la importancia de la información en un contexto económico. González (2002), por ejemplo, señala que la información es conocimiento codificado y es parte de la producción tanto material como intelectual de la sociedad, ya que conocerla y divulgarla permite coordinar esfuerzos y sincronizar acciones para la toma de decisiones. Adicionalmente, este autor afirma que las nuevas reglas de los mercados están caracterizadas por los paradigmas de las tecnologías de la información, por la producción y la aplicación intensiva del conocimiento y la tecnología a los negocios, todo con una dinámica acelerada de expansión de Internet como una red pública abierta.

En este sentido, juega un papel fundamental el uso de las tecnologías en la comunicación de información financiera, y es, por tanto, este el objeto de estudio desarrollado en la presente investigación, en razón a que se analiza la divulgación de los estados financieros de la organizaciones que forman parte del sector solidario. Lo anterior nos permite entender cómo las herramientas de tecnología, información y comunicación son poco aprovechadas en las entidades del sector privado, ya que su uso limitado y el desconocimiento de los autores sobre la información financiera que debe ser pública disminuyen los índices de transparencia que pueden alcanzar estas entidades.

En el documento se abordan, inicialmente, los aspectos metodológicos con respecto al índice de medición planteado en la investigación cuantitativa que se desarrolla en este documento. Posteriormente, se presentan los resultados de la investigación, los cuales se acompañan de las conclusiones derivadas del estudio realizado; al documento se adjuntan también los anexos en los que se identifican las entidades que conformaron el estudio.

\section{Metodología}

El uso de las TIC facilita la divulgación de información financiera y mejora los niveles de transparencia en las organizaciones que los emplean. Algunas de las entidades con mayor grado de interés del público por la estructura de su patrimonio son las entidades que forman parte de la economía solidaria y se encuentran vigiladas por la Superintendencia de Economía Solidaria.

Es por eso que la población objetivo de esta investigación está conformada por las entidades vigiladas por la Superintendencia de Economía Solidaria que posean activos superiores a 20000 millones de pesos con corte a 31 de diciembre del 2016, y cuenten en su planta de personal con más de 10 empleados. Estas entidades se seleccionaron considerando que son las de mayor capacidad financiera y, por tanto, es viable que tengan página web para interactuar con sus usuarios.

Del total de entidades vigiladas por la Superintendencia de Economía Solidaria y que cumplen las condiciones establecidas, se encontraron 222 en total. De este número de entidades, se seleccionó una muestra que abarca al $50 \%$ del total de empresas que cumplen los criterios de selección (para estas organizaciones se realizó inicialmente la búsqueda de páginas web en las que se divulga la información de la entidad). 
A fin de analizar los factores de divulgación se desarrolló un índice de medición que evalúa el porcentaje de cumplimiento frente a la presentación de estados financieros por medio de herramientas TIC, como, por ejemplo, las páginas de Internet de las entidades analizadas. Este índice de medición lo componen 10 aspectos de análisis que se presentan en la tabla 1.

TABLA 1. Índice de cumplimiento (Ic)

\begin{tabular}{|c|c|}
\hline ASPECTO EVALUADO & PORCENTAJE \\
\hline a. La entidad tiene página web & $10 \%$ \\
\hline $\begin{array}{l}\text { b. Existe una sección de información } \\
\text { financiera en la página web de la } \\
\text { entidad }\end{array}$ & $10 \%$ \\
\hline $\begin{array}{l}\text { c. La entidad presenta información } \\
\text { referente a su estado de situación } \\
\text { financiera }\end{array}$ & $10 \%$ \\
\hline $\begin{array}{l}\text { d. La entidad presenta información } \\
\text { referente a su estado de resultados }\end{array}$ & $10 \%$ \\
\hline $\begin{array}{l}\text { e. La entidad presenta información } \\
\text { referente a su estado de cambios en el } \\
\text { patrimonio }\end{array}$ & $10 \%$ \\
\hline $\begin{array}{l}\text { f. La entidad presenta información } \\
\text { referente a su estado de flujo de } \\
\text { efectivo }\end{array}$ & $10 \%$ \\
\hline $\begin{array}{l}\text { g. La entidad presenta información } \\
\text { referente a las notas a los estados } \\
\text { financieros }\end{array}$ & $10 \%$ \\
\hline $\begin{array}{l}\text { h. Se permite el acceso libre de la } \\
\text { información financiera para los usuarios }\end{array}$ & $10 \%$ \\
\hline i. La información presentada es legible & $10 \%$ \\
\hline $\begin{array}{l}\text { j. La información financiera se presenta } \\
\text { de forma comparativa de un año a otro }\end{array}$ & $10 \%$ \\
\hline Total & $100 \%$ \\
\hline
\end{tabular}

Elaboración propia

Para la medición de estos factores se aplicó un sistema de puntuación dicotómica, que ha sido empleado en otras investigaciones por autores como Pérez y López (2003). A través de este sistema se asigna una puntuación de uno cuando se cumple con la condición evaluada, y cero si la entidad objeto de estudio no cumple con la condición requerida. En este sentido, el índice de cumplimiento se mide al sumar los aspectos que cumplen con la condición evaluada y dividiéndolos por el total de ítems evaluados.

Dado lo anterior, el índice de cumplimiento para los factores analizados estaría representado por:

$$
I C=\frac{\sum \quad \begin{array}{c}
\text { Item efectivamente } \\
\text { presentado }
\end{array}}{\text { Número de ítems a evaluar }}
$$

Con esta medición es posible determinar el grado de cumplimiento de las entidades objeto de estudio frente a los aspectos mínimos de divulgación de la información financiera, precisando que para cada uno de los componentes analizados no se estudian los criterios de revelación de las NIIF aplicados en cada estado financiero, ya que dicha información podría abarcar un nuevo estudio. En este caso el objetivo primordial es identificar si las entidades analizadas divulgan sus estados financieros con el empleo de herramientas de tecnología de la información y la comunicación.

\section{Divulgación de información financiera en entidades privadas del sector solidario con el empleo de herramientas TIC}

Uno de los beneficios principales asociados a la adopción de normas internacionales de información financiera (NIIF) está relacionado con la toma de decisiones y los elementos de comparabilidad que trae la norma internacional, lo cual facilita el análisis de información por parte de los usuarios. Bajo estos parámetros se considera que la información financiera presentada en los estados financieros es pública, con el fin de que puedan conocerla los diferentes grupos de intereses que existen alrededor de ella.

Existen diferentes mecanismos por medio de los cuales un usuario puede acceder a la información financiera. Uno de estos es el empleo de TIC, entendiéndose estas como las herramientas, los soportes y los canales computacionales e informáticos que, entre otras funciones, sirven para la difusión de información de las compañías y como mecanismo de comunicación entre los usuarios (Duran, 2015).

Una de las principales herramientas empleadas para difusión de la información financiera, asociada al uso de las TIC, se da por medio de las conexiones a Internet, ya que estas juegan un papel fundamental en cuanto instrumento que permite acceder a información financiera de diferentes entes gubernamentales y compañías privadas, pues son de rápido y fácil acceso para los usuarios de la información (Peña, Vargas \& Medina, 2012). 
Diferentes estudios han analizado la difusión de información financiera por parte de las entidades gubernamentales en América Latina. Gómez y Montesinos (2014) analizan la trasparencia en las entidades gubernamentales por medio de herramientas como el Gobierno electrónico, un instrumento de modernización de la administración pública que mejora la transparencia y la divulgación de la información financiera y presupuestal de las entidades públicas en Colombia.

La difusión y comparabilidad de la información económico-financiera on-line ha sido estudiada también por Peña, Vargas \& Medina (2012), quienes realizan un ranking de los países de Iberoamérica más transparentes al divulgar información económico-financiera, y resaltan la importancia del uso de las TIC para mejorar los niveles de transparencia en las entidades objeto de análisis.

Sin embargo, las publicaciones asociadas a la divulgación de información financiera de entidades en el sector privado con el empleo de mecanismos asociados a las TIC - como lo es el uso de Internet- son nulas. Por tanto, en el propósito de atender este vacío, en esta investigación se analizaron las páginas de Internet de 110 entidades que conforman el sector solidario, con el fin de buscar información referente a los estados financieros de estas compañías e identificar cuáles de ellas emplean este mecanismo como difusor de su información financiera.

Las entidades del sector solidario representan una fuerza importante dentro de la economía nacional. Uno de los principios de las entidades que conforman el sector solidario es que desarrollan su actividad sin ánimo de lucro, por tanto, no existe una distribución de excedentes si se considera, adicionalmente, que el patrimonio de estas entidades lo conforman, en gran medida, los aportes realizados por sus asociados (Rendón, Montaño \& Gaitán, 2011).

El análisis realizado sobre divulgación de información financiera para aquellas entidades vigiladas por la Superintendencia Solidaria comprendió 10 aspectos básicos, los cuales buscan identificar si las entidades privadas divulgan sus estados financieros con el empleo de herramientas TIC, como lo es permitir el acceso a la información presentada en los estados financieros de la entidad por medio de su página de Internet, y que esta sea legible y se muestre comparable de un periodo a otro, aspectos que se presentaron en la sección metodológica de este documento.
En este análisis observamos que de las 110 entidades objeto de estudio, un $96 \%$ de ellas cuentan con página web. En dichas páginas se observó que apenas un 29 \% cuenta con una sección en la que se revela información financiera, y tan solo un $22 \%$ de estas presenta información relacionada con los estados financieros de la entidad.

Respecto a los estados financieros presentados se observó que las entidades no presentan un juego completo de estados financieros requeridos, tal y como lo indica el Decreto 2420 de 2015, por el cual se establece que se debe presentar como mínimo el estado de situación financiera, el estado de resultados, el estado de flujo de efectivo, el estado de cambios en el patrimonio y las notas a los estados financieros, ya que si bien un $22 \%$ de las entidades analizadas presenta información relacionada con el estado de situación financiera, tan solo un $21 \%$ presenta información asociada al estado de resultados.

La situación frente al estado de flujo de efectivo y el estado de cambios en el patrimonio evidencia la menor importancia que se da frente a la divulgación de estos estados financieros, ya que apenas un $19 \%$ de las entidades analizadas presenta dicha información en sus páginas web. Esto muestra cómo aunque algunas entidades presentan el estado de situación financiera, no pasa lo mismo con el de resto de estados financieros requeridos para una toma de decisiones eficaz.

Una situación similar se presenta con las notas a los estados financieros, ya que tan solo un $18 \%$ de las entidades analizadas revelan información en su página web asociada con estos conceptos. Esto es un asunto de particular importancia, ya que las notas a los estados financieros resultan fundamentales para conocer los alcances de las cifras que se presentan al interior de los estados financieros, con lo cual los usuarios de la información pueden alcanzar un mayor entendimiento de la información presentada.

En relación con el libre acceso a la información financiera, del $22 \%$ de entidades que presentan información relacionada con sus estados financieros, un $91 \%$ permite acceder de forma libre, mientras que el $9 \%$ restante permite el acceso solo para asociados de la entidad analizada. Al consultar con las entidades que restringen la información, con respecto a las razones de las limitaciones en el acceso estas informan que la información está disponible únicamente para los asociados, ya que la consideran información de 
carácter privado y que esta no se puede compartir con terceros.

En cuanto a la comprensibilidad inicial de los estados financieros publicados en la página web de cada una de las entidades analizadas, un $87,5 \%$ es legible, puesto que la información restante se muestra en presentaciones o por medio de aplicaciones que no son legibles. Por tanto, la información presentada no pueden emplearla los usuarios para la toma de decisiones, dado que es imposible realizar una lectura sobre los datos presentados.

Una de las características fundamentales de la información financiera, conforme a los parámetros establecidos en el marco conceptual de las NIIF, es que la información debe presentarse de forma comparativa de un año a otro, situación que se cumple en el $91 \%$ de las entidades que divulgan información con el empleo de sus páginas web. En el $9 \%$ de entidades restantes la información no se presenta de forma comparativa, aspecto que puede afectar la toma de decisiones si se considera que no es posible realizar un análisis de comportamiento o de variaciones cuando no se cuenta con información comparable.

Con respecto al total de entidades analizadas, tan solo un $11 \%$ de estas cumple con todos los aspectos de medición establecidos en la investigación, mientras que un $64 \%$ de las entidades objetivo de estudio cumplen con menos de dos criterios, lo cual evidencia la escasa divulgación de información en las entidades objeto de estudio.

\section{Conclusiones}

Al analizar los resultados observados frente a la divulgación de información financiera de entidades del sector solidario, es posible afirmar que existe poca divulgación de los estados financieros de las compañías con el empleo de herramientas TIC - como lo es el uso de Internet- que faciliten el acceso a la información financiera. Esta situación, como lo indican Católico, Cely \& Pulido (2013), puede generar problemas de asimetría de la información, los cuales se presentan cuando se omite información requerida por los grupos de interés (stakeholders).

Frente a las entidades que divulgan información, un alto porcentaje de estas lo hace sin el cumplimiento de los requisitos mínimos para realizar dicha labor, lo cual dificulta la toma de decisiones por parte de los usuarios. En este caso no se puede obviar que la información financiera y el uso de las Tic están fuertemente relacionados con las actividades de globalización y homologación de la información financiera, de manera que es este uno de los objetivos principales que se espera alcanzar en los procesos de adopción de NIIF. En este sentido, como lo afirma Duran (2015), la información financiera debe tener estándares de calidad que contribuyan a la toma de decisiones por parte de los usuarios, situación que no se presenta en el $88 \%$ de los casos analizados.

Uno de los aspectos a considerar en los resultados de la investigación es el hecho de que tan solo un $22 \%$ de las entidades analizadas permite acceder a la información financiera, y que de ese grupo de entidades exista un $9 \%$ que no permita el acceso de la información para todos sus usuarios, de modo que restringe el acceso para los asociados actuales de la entidad. Este es un aspecto a evaluar no solo por las implicaciones normativas inmersas en la obligación de publicar información financiera, sino en razón a que las entidades potenciarían su actividad si permitieran que futuros interesados - como lo es el caso de asociados con interés de vincularse a la entidad- pudieran conocer la información presentada en los estados financieros.

Otro de los aspectos que llama especialmente la atención es la poca importancia otorgada a la divulgación de las notas a los estados financieros, si se considera que estas solo se publicaron en un $18 \%$ de los casos analizados. En este sentido, es importante recordar que la revelación de información financiera que acompaña los estados financieros es fundamental para una correcta toma de decisiones por parte de los usuarios, ya que allí se presentan aspectos ignorados o que no pueden ser valorados en la presentación de cada uno de los estados financieros, y que son necesarios para comprender y evaluar los hechos económicos de las entidades.

Al respecto es importante precisar - tal y como lo indican Arias, Portilla \& Agredo (2007) que la información contable difundida de manera errada puede distorsionar lo que realmente ocurre en las entidades, y llevar así a la toma de decisiones erradas para la vinculación de un tercero con la entidad de la cual se pretende analizar la información financiera. Por esto, la importancia de que las entidades presenten la información financiera, que incluya toda la información requerida que forma un juego completo de estados financieros. 
En este proceso de revelación de la información uno de los aspectos fundamentales es que la información sea comparable y de un año a otro puedan observarse las variaciones asociadas a los movimientos en los hechos económicos, aspectos que no se presentan en el $9 \%$ de las entidades analizadas, por tanto en estos casos no se estarían cumpliendo con algunos de los requerimientos de las normas NIIF frente a la información presentada, ya que allí se definen las características cualitativas de mejora de la información, las cuales abarcan aspectos tales como la comparabilidad, la verificabilidad, la oportunidad y la comprensibilidad de la información.

Con los resultados obtenidos en la investigación es posible afirmar que existe un camino largo para que las entidades del sector privado, y en este caso en particular las entidades que forman parte de la economía solidaria, divulguen la información de sus estados financieros por medio de herramientas TIC que favorezcan la relación entre la entidad y el usuario. El desarrollo de este campo es fundamental si se considera que la información financiera debe ser libre, debe mejorar los niveles de transparencia y servir para la toma de decisiones, mientras que las restricciones sobre la información financiera dificultan los procesos de globalización y desaceleran el crecimiento económico de las entidades.

Finalmente, en la búsqueda de información requerida para esta investigación y en las respuestas obtenidas por las entidades consultadas se evidenció que existe cierto grado de desconocimiento respecto a la información financiera de carácter público que normativamente debe ser divulgada, por lo cual en este campo surge un nuevo interrogante que puede abordarse en futuras investigaciones.

\section{REFERENCIAS}

Arias, M., Portilla, L., y Agredo, L. (2007, diciembre). La información contable en el análisis de los estados financieros. Revista Scientia et Técnica, XIII(37), 311-314.

Católico, D., Cely, A., y Pulido J., (2013). Revelación de la información financiera sobre propiedad planta y equipo, en empresas industriales cotizadas en Colombia. Revista Cuadernos de Contabilidad, 943970.

Cooperativa de Ahorro y Crédito Berlín. (2017). Estados financieros a diciembre de 2016. Recuperado de https://www.invercoob.com/
Cooperativa de Ahorro y Crédito Fincomercio Ltda. (2017). Estados financieros a diciembre de 2016. Recuperado de https://www.fincomercio.com/ Cooperativa de Ahorro y Crédito Serviunal. (2017). Estados financieros a diciembre de 2016. Recuperado de www.cooservunal.com

Cooperativa de Trabajadores de Bavaria Dirección y Ventas Ltda. (2017). Estados financieros a diciembre de 2016. Recuperado de http://badivencoop. coop/

Cooperativa de Profesionales Sanitas. (2017). Estados financieros a diciembre de 2016. Recuperado de http://www.cooperativasanitas.com/

Cooperativa Multiactiva Empresarial Coovitel. (2017). Estados financieros a diciembre de 2016. Recuperado de http://www.coovitel.coop/

Cooperativa Nacional del Sector de las Comunicaciones y Entidades Afines y Relacionadas Ltda. (2017). Estados financieros a diciembre 31 de 2016. Recuperado de http://coopmincom.com/

Cooperativa Nacional de Trabajadores-Coopetraban. (2017). Estados financieros a diciembre 31 de 2016. Recuperado de http://www.coopetraban.com.co/

Cooperativa de Empleados y Obreros del Departamento de Caldas. (2017). Estados financieros a diciembre de 2016. Recuperado de https://www. ceocal.co/

Cooperativa de Trabajadores y Pensionados de la E. A. A. B.-Cooacueducto. (2017). Estados financieros a 31 de diciembre de 2016. Recuperado de http:// www.cooacueducto.coop/

Cooperativa Multiactiva Universitaria Nacional. (2017). Estados financieros a diciembre de 2016. Recuperado de http://www.comuna.com.co/

Decreto 2420. Por medio del cual se expide el Decreto Único Reglamentario de las Normas de Contabilidad, de Información Financiera y de Aseguramiento de la Información y se dictan otras disposiciones.

Duran, Y. (2013). La contabilidad internacional un enfoque para la presentación de información financiera. Revista del Centro de Investigación. Universidad de la Salle, 10(39), 33-56.

Duran, Y. (2015, enero-junio). Contabilidad bajo el enfoque de tecnologías de la información y comunicación (TIC's). Estudio basado en la pyme del sector salud privado, municipio Valera estado Trujillo Venezuela. Revista Visión Gerencial, 1, 53-88.

Febor Entidad Cooperativa. (2017). Estados financieros a diciembre de 2016. Recuperado de http://www. febor.coop/

Fondo de Beneficio Común de los Empleados del Sena. (2017). Estados financieros a diciembre de 2016. Recuperado de http://www.fbcsena.com/

Fondo de Docentes Universidad del Valle. (2017). Estados financieros a diciembre de 2016. Recuperado de www.fonvalle.com 
Fondo de Empleados Coomeva. (2017). Estados financieros a diciembre de 2016. Recuperado de http:// fecoomeva.coomeva.com.co/

Fondo de empleados BBvA Colombia. (2017). Estados financieros a diciembre de 2016. Recuperado de https://www.foebbva.com/

Fondo de Empleados de Colsanitas. (2017). Estados financieros a diciembre de 2016. Recuperado de http://www.fecolsa.com.co/

Fondo de Empelados del Cerrejón. (2017). Estados financieros a diciembre de 2016. Recuperado de http://www.fondecor.org.co/

Fondo de empelados del ciat. (2017). Estados financieros a diciembre de 2016. Recuperado de www. creciat.com.co

Fondo de Empleados de Johnson y Johnson de Colombia Ltda. (2017). Estados financieros a diciembre de 2016. Recuperado de www.fedejohnson.com

Fondo de Empleados del Grupo Bancolombia. (2017). Estados financieros a diciembre de 2016. Recuperado de https://www.febanc.com.co/

Fondo de Empleados y Pensionados de la EтB-Fontebo. Estados financieros a diciembre de 2016. Recuperado de https://www.fontebo.com/
Fondo de Empleados para Bienestar Funcionarios del ICBF. (2017). Estados financieros a diciembre de 2016. Recuperado de http://www.fonbienestar. com.co

Gómez, V., y Montesinos J., (2014). Gobierno electrónico y transparencia financiera y presupuestal de los departamentos en Colombia. Revista Venezolana de Gerencia, 19(68), 670-698.

Peña, J., Vargas, C., y Medina I., (2012, junio). Difusión y comparabilidad de la información económica-financiera on-line: el caso de Iberoamérica. Revista facultad de ciencias económicas, $X X, 231-245$.

Pérez, C., y López, A. (2003). La difusión de información financiera gubernamental en los países del Mercosur: su amortización a través de la aplicación de Ipsas de la IFAC. Revista Contabilidad y Finanzas-USP, 14(33), 90-100.

Rendón, B., Montaño, E., y Gaitán, G., (2011). Las NIIF y su impacto en las cooperativas en Colombia a diciembre 31 de 2011. Revista Cuadernos de Administración, 29(50), 176-185. 


\section{Apéndice A. Entidades analizadas}

\begin{tabular}{|c|c|c|c|c|c|c|c|c|c|c|c|}
\hline NIT & NOMBRE DE LA ENTIDAD-SIGLA & A & B & C & D & $\mathrm{E}$ & $\mathrm{F}$ & G & $\mathrm{H}$ & I & $\mathrm{J}$ \\
\hline $800-005-340-4$ & Fondo de Empleados de Coomeva & 1 & 1 & 1 & 1 & 1 & 1 & 1 & 1 & 1 & 1 \\
\hline $800-020-684-5$ & $\begin{array}{l}\text { Cooperativa de Ahorro y Crédito Nacional } \\
\text { Limitada }\end{array}$ & 1 & 0 & 0 & 0 & 0 & 0 & 0 & 0 & 0 & 0 \\
\hline $800-025-304-4$ & $\begin{array}{l}\text { Fondo de Empleados Empresas Públicas de } \\
\text { Medellín-Fepep) }\end{array}$ & 1 & 0 & 0 & 0 & 0 & 0 & 0 & 0 & 0 & 0 \\
\hline $800-052-963-2$ & $\begin{array}{l}\text { Fondo Empleados para Bienestar } \\
\text { Funcionarios del-ICBF }\end{array}$ & 1 & 1 & 1 & 1 & 1 & 1 & 1 & 1 & 1 & 1 \\
\hline $800-069-695-8$ & $\begin{array}{l}\text { Fondo de Empleados de la Comunidad } \\
\text { Educativa del Ariari }\end{array}$ & 1 & 1 & 0 & 0 & 0 & 0 & 0 & 0 & 0 & 0 \\
\hline $800-069-925-7$ & Cooperativa Financiera Cafetera & 1 & 0 & 0 & 0 & 0 & 0 & 0 & 0 & 0 & 0 \\
\hline $800-077-762-7$ & $\begin{array}{l}\text { Cooperativa de Trabajadores de Bavaria } \\
\text { Dirección y Ventas Ltda. }\end{array}$ & 1 & 1 & 1 & 1 & 1 & 1 & 1 & 1 & 1 & 1 \\
\hline $800-097-913-8$ & Fondo de Empleados de Granfondo & 1 & 1 & 0 & 0 & 0 & 0 & 0 & 0 & 0 & 0 \\
\hline $800-112-808-7$ & $\begin{array}{l}\text { Fondo de Empleados Docentes de la } \\
\text { Universidad Nal. }\end{array}$ & 1 & 0 & 0 & 0 & 0 & 0 & 0 & 0 & 0 & 0 \\
\hline $800-183-987-0$ & Fondo de Empleados Almacenes Éxito & 1 & 0 & 0 & 0 & 0 & 0 & 0 & 0 & 0 & 0 \\
\hline 800-201-989-3 & $\begin{array}{l}\text { Cooperativa de Ahorro y Crédito Creafam- } \\
\text { Coocreafam }\end{array}$ & 1 & 0 & 0 & 0 & 0 & 0 & 0 & 0 & 0 & 0 \\
\hline $804-009-752-8$ & $\begin{array}{l}\text { Cooperativa de Ahorro y Crédito de } \\
\text { Santander Limitada-Financiera Comultrasan } \\
\text { Ltda. }\end{array}$ & 1 & 0 & 0 & 0 & 0 & 0 & 0 & 0 & 0 & 0 \\
\hline $805-004-034-9$ & $\begin{array}{l}\text { Cooperativa de Servidores Públicos \& } \\
\text { Jubilados de Colombia-Coopserp Colombia }\end{array}$ & 1 & 0 & 0 & 0 & 0 & 0 & 0 & 0 & 0 & 0 \\
\hline $805-019-569-2$ & $\begin{array}{l}\text { Distribuidora Nacional Cooperativa } \\
\text { Multiactiva }\end{array}$ & 0 & 0 & 0 & 0 & 0 & 0 & 0 & 0 & 0 & 0 \\
\hline $830-033-907-8$ & $\begin{array}{l}\text { Progressa entidad cooperativa de ahorro y } \\
\text { crédito }\end{array}$ & 1 & 1 & 0 & 0 & 0 & 0 & 0 & 0 & 0 & 0 \\
\hline $830-113-916-8$ & Cooperativa de Profesionales Sanitas & 1 & 0 & 1 & 1 & 1 & 1 & 1 & 1 & 0 & 1 \\
\hline $860-005-921-1$ & Casa Nacional del Profesor S.C.I.-Canapro & 1 & 1 & 0 & 0 & 0 & 0 & 0 & 0 & 0 & 0 \\
\hline $860-007-266-4$ & $\begin{array}{l}\text { Fondo de Empleados del Grupo Seguros } \\
\text { Bolivar-Adebol }\end{array}$ & 1 & 0 & 0 & 0 & 0 & 0 & 0 & 0 & 0 & 0 \\
\hline $860-007-327-5$ & $\begin{array}{l}\text { Cooperativa de Ahorro y Crédito Fincomercio } \\
\text { Ltda.-Fincomercio Itda }\end{array}$ & 1 & 1 & 1 & 1 & 1 & 1 & 1 & 1 & 1 & 1 \\
\hline $860-007-647-7$ & Febor Entidad Cooperativa & 1 & 1 & 1 & 1 & 1 & 1 & 1 & 1 & 1 & 1 \\
\hline $860-007-783-0$ & Asociacion Mutual Corfeinco & 1 & 0 & 0 & 0 & 0 & 0 & 0 & 0 & 0 & 0 \\
\hline $860-011-265-2$ & Fondo de Empleados BBva Colombia & 1 & 1 & 1 & 1 & 1 & 1 & 1 & 1 & 1 & 1 \\
\hline $860-013-717-9$ & Caja Cooperativa Credicoop & 1 & 0 & 0 & 0 & 0 & 0 & 0 & 0 & 0 & 0 \\
\hline $860-013-743-0$ & Caja Cooperativa Petrolera & 1 & 0 & 0 & 0 & 0 & 0 & 1 & 0 & 0 & 0 \\
\hline $860-014-040-6$ & $\begin{array}{l}\text { Cooperativa de los Profesionales de la Salud } \\
\text { Coasmedas }\end{array}$ & 1 & 0 & 0 & 0 & 0 & 0 & 0 & 0 & 0 & 0 \\
\hline $860-014-397-1$ & $\begin{array}{l}\text { Cooperativa de los Trabajadores del } \\
\text { Instituto de los Seguros Sociales }\end{array}$ & 1 & 0 & 0 & 0 & 0 & 0 & 0 & 0 & 0 & 0 \\
\hline $860-015-017-0$ & $\begin{array}{l}\text { Cooperativa Multiactiva Empresarial } \\
\text { Coovitel }\end{array}$ & 1 & 0 & 1 & 1 & 1 & 1 & 1 & 1 & 1 & 1 \\
\hline $860-015-964-0$ & $\begin{array}{l}\text { Cooperativa Nacional del Sector de las } \\
\text { Comunicaciones y Entidades Afines y } \\
\text { Relacionadas Ltda. }\end{array}$ & 1 & 0 & 1 & 1 & 1 & 1 & 1 & 1 & 1 & 1 \\
\hline
\end{tabular}




\begin{tabular}{|c|c|c|c|c|c|c|c|c|c|c|c|}
\hline NIT & NOMBRE DE LA ENTIDAD-SIGLA & A & B & C & D & $\mathrm{E}$ & $\mathrm{F}$ & G & H & I & $J$ \\
\hline $860-017-111-4$ & Cooperativa Texas Ltda. & 1 & 0 & 0 & 0 & 0 & 0 & 0 & 0 & 0 & 0 \\
\hline $860-021-738-7$ & $\begin{array}{l}\text { Cooperativa de Trabajadores y Pensionados } \\
\text { de la E.A.A.B.-Cooacueducto }\end{array}$ & 1 & 1 & 1 & 1 & 1 & 1 & 0 & 1 & 1 & 1 \\
\hline $860-023-189-2$ & $\begin{array}{l}\text { Cooperativa de Ahorro y Crédito de } \\
\text { Trabajadores de Peldar y otros de Colombia }\end{array}$ & 1 & 0 & 0 & 0 & 0 & 0 & 0 & 0 & 0 & 0 \\
\hline $860-026-153-1$ & $\begin{array}{l}\text { Cooperativa Multiactiva de Profesionales } \\
\text { Somec }\end{array}$ & 1 & 1 & 0 & 0 & 0 & 0 & 0 & 0 & 0 & 0 \\
\hline $860-027-186-9$ & $\begin{array}{l}\text { Cooperativa de Profesores de la U. Nacional } \\
\text { de Colombia }\end{array}$ & 1 & 0 & 0 & 0 & 0 & 0 & 0 & 0 & 0 & 0 \\
\hline $860-029-924-7$ & Universidad Cooperativa de Colombia (UCC) & 1 & 0 & 0 & 0 & 0 & 0 & 0 & 0 & 0 & 0 \\
\hline $860-032-342-1$ & $\begin{array}{l}\text { Cooperativa de Ahorro y Crédito de Tenjo- } \\
\text { Cooptenjo }\end{array}$ & 1 & 0 & 0 & 0 & 0 & 0 & 0 & 0 & 0 & 0 \\
\hline $860-033-227-7$ & $\begin{array}{l}\text { Cooperativa Empresarial Multiactiva } \\
\text { Popular-Coempopular }\end{array}$ & 1 & 0 & 0 & 0 & 0 & 0 & 0 & 0 & 0 & 0 \\
\hline $860-035-559-6$ & Fondo de Empleados Feisa-Feisa & 1 & 0 & 0 & 0 & 0 & 0 & 0 & 0 & 0 & o \\
\hline $860-040-212-6$ & $\begin{array}{l}\text { Fondo de empleados y pensionados de la } \\
\text { ETB-Fontebo }\end{array}$ & 1 & 1 & 1 & 1 & 0 & 0 & 0 & 1 & 1 & 1 \\
\hline $860-047-066-9$ & $\begin{array}{l}\text { Cooperativa de Impresores y Papeleros de } \\
\text { Bogotá }\end{array}$ & 1 & 1 & 0 & 0 & 0 & 0 & 0 & 0 & 0 & 0 \\
\hline $860-048-092-5$ & $\begin{array}{l}\text { Fondo de Empleados de Davivienda- } \\
\text { Fondavivienda }\end{array}$ & 1 & 0 & 0 & 0 & 0 & 0 & 0 & 0 & 0 & 0 \\
\hline $860-048-537-0$ & $\begin{array}{l}\text { Corporacion Fondo de Empleados para } \\
\text { Vivienda del Instituto de Seguros Sociales y } \\
\text { demás Entidades de la Seguridad Social }\end{array}$ & 1 & 0 & 0 & 0 & 0 & 0 & 0 & 0 & 0 & 0 \\
\hline $860-049-363-0$ & $\begin{array}{l}\text { Cooperativa de empleados de Cafam- } \\
\text { Coopcafam }\end{array}$ & 1 & 0 & 0 & 0 & 0 & 0 & 0 & 0 & 0 & 0 \\
\hline $860-056-869-4$ & Cooperativa de Ahorro y Crédito Crediflores & 1 & 0 & 0 & 0 & 0 & 0 & 0 & 0 & 0 & o \\
\hline $860-068-522-6$ & Cooperativa de Empleados de Dow Colombia & 1 & 0 & 0 & 0 & 0 & 0 & 0 & 0 & 0 & 0 \\
\hline $860-075-780-9$ & Cooperativa del Sistema Nacional de Justicia & 1 & 1 & 1 & 0 & 0 & 0 & 0 & 0 & 0 & 0 \\
\hline $860-402-925-3$ & $\begin{array}{l}\text { Cooperativa Multiactiva de los Trabajadores } \\
\text { de la Educación de cundinamarca y el } \\
\text { Distrito Capital-Cootradecun }\end{array}$ & 1 & 0 & 0 & 0 & 0 & 0 & 0 & 0 & 0 & 0 \\
\hline $860-514-823-1$ & Cooperativa para el Bienestar Social & 1 & 0 & 0 & 0 & 0 & 0 & 0 & 0 & 0 & o \\
\hline $860-516-881-8$ & Central Cooperativa de Servicios Funerarios & 1 & 0 & 0 & 0 & 0 & 0 & 0 & 0 & 0 & 0 \\
\hline $860-518-350-8$ & $\begin{array}{l}\text { Cooperativa de Ahorro y Crédito para el } \\
\text { Bienestar Social- Beneficiar }\end{array}$ & 1 & 0 & 0 & 0 & 0 & 0 & 0 & 0 & 0 & 0 \\
\hline $860-527-467-9$ & Fondo de Empleados de Colsanitas & 1 & 1 & 1 & 1 & 1 & 1 & 1 & 1 & 1 & 1 \\
\hline $860-534-049-2$ & Fondo de Empleados de Colsubsidio & 1 & 1 & 0 & 0 & 0 & 0 & 0 & 0 & 0 & 0 \\
\hline $890-002-377-1$ & $\begin{array}{l}\text { Cooperativa Nacional de Ahorro y Crédito } \\
\text { Avanza }\end{array}$ & 1 & 0 & 0 & 0 & 0 & 0 & 0 & 0 & 0 & 0 \\
\hline $890-112-491-3$ & Fondo de Empleados del Cerrejón & 1 & 1 & 1 & 1 & 1 & 1 & 1 & 1 & 1 & 1 \\
\hline $890-201-063-6$ & $\begin{array}{l}\text { Cooperativa Multiactiva de los Trabajadores } \\
\text { de Santander- Coomultrasan }\end{array}$ & 1 & 0 & 0 & 0 & 0 & 0 & 0 & 0 & 0 & 0 \\
\hline $890-201-280-8$ & $\begin{array}{l}\text { Cooperativa de Ahorro y Crédito de } \\
\text { Profesores }\end{array}$ & 1 & 1 & 0 & 0 & 0 & 0 & 0 & 0 & 0 & 0 \\
\hline $890-203-225-1$ & $\begin{array}{l}\text { Cooperativa de Ahorro y Créedito para } \\
\text { el Desarrollo Solidario de Colombia- } \\
\text { Coomuldesa Ltda. }\end{array}$ & 1 & 0 & 0 & 0 & 0 & 0 & 0 & 0 & 0 & 0 \\
\hline
\end{tabular}




\begin{tabular}{|c|c|c|c|c|c|c|c|c|c|c|c|}
\hline NIT & NOMBRE DE LA ENTIDAD-SIGLA & A & B & C & D & $\mathrm{E}$ & $\mathrm{F}$ & G & $\mathrm{H}$ & I & J \\
\hline $890-203-827-5$ & $\begin{array}{l}\text { Cooperativa de Servicios Múltiples de la } \\
\text { Provincia de Vélez Ltda. }\end{array}$ & 1 & 0 & 0 & 0 & 0 & 0 & 0 & 0 & 0 & 0 \\
\hline $890-204-348-3$ & Cooperativa Multiservicios Barichara Ltda. & 1 & 1 & 0 & 0 & 0 & 0 & 0 & 0 & 0 & 0 \\
\hline $890-270-045-8$ & $\begin{array}{l}\text { Cooperativa de Ahorro y Crédito Financiera } \\
\text { Coagrosur. }\end{array}$ & 1 & 0 & 0 & 0 & 0 & 0 & 0 & 0 & 0 & 0 \\
\hline $890-303-400-3$ & Cooperativa de Ahorro y Crédito Berlín. & 1 & 0 & 1 & 1 & 0 & 0 & 1 & 1 & 0 & 1 \\
\hline $890-303-438-2$ & Multiactiva El Roble, Entidad Cooperativa & 1 & 0 & 0 & 0 & 0 & 0 & 0 & 0 & 0 & 0 \\
\hline $890-308-458-2$ & $\begin{array}{l}\text { Fondo de Empleados del Banco de } \\
\text { Occidente- Fondoccidente }\end{array}$ & 1 & 0 & 0 & 0 & 0 & 0 & 0 & 0 & 0 & 0 \\
\hline 890-308-934-7 & Fondo de Empleados del CIAT & 1 & 0 & 1 & 1 & 1 & 1 & 1 & 1 & 1 & 1 \\
\hline $890-317-598-3$ & $\begin{array}{l}\text { Fondo de Empleados Docentes Universidad } \\
\text { del Valle }\end{array}$ & 1 & 1 & 1 & 1 & 1 & 1 & 1 & 1 & 1 & 1 \\
\hline $890-318-786-6$ & $\begin{array}{l}\text { Fondo de Empleados de Johnson \& Johnson } \\
\text { de Colombia }\end{array}$ & 1 & 0 & 1 & 1 & 1 & 1 & 1 & 1 & 1 & 1 \\
\hline $890-401-658-6$ & $\begin{array}{l}\text { Cooperativa de Educadores y Empleados de } \\
\text { la Educación- Cooaceded Ltda. }\end{array}$ & 1 & 0 & 0 & 0 & 0 & 0 & 0 & 0 & 0 & 0 \\
\hline $890-500-571-9$ & $\begin{array}{l}\text { Cooperativa Agropecuaria del Norte de } \\
\text { Santander }\end{array}$ & 1 & 0 & 0 & 0 & 0 & 0 & 0 & 0 & 0 & 0 \\
\hline $890-505-363-6$ & $\begin{array}{l}\text { Cooperativa Especializada de Ahorro y } \\
\text { Crédito Crediservir- Crediservir }\end{array}$ & 1 & 0 & 0 & 0 & 0 & 0 & 0 & 0 & 0 & 0 \\
\hline $890-700-817-3$ & $\begin{array}{l}\text { Cooperativa de Maestros y Empleados de la } \\
\text { Educación del Tolima-Coopemtol }\end{array}$ & 1 & 0 & 0 & 0 & 0 & 0 & 0 & 0 & 0 & 0 \\
\hline $890-701-355-7$ & Cooperativa Serviarroz Ltda. & 1 & 0 & 0 & 0 & 0 & 0 & 0 & 0 & 0 & 0 \\
\hline $890-802-543-9$ & $\begin{array}{l}\text { Cooperativa de Empleados y Obreros del } \\
\text { Departamento de Caldas }\end{array}$ & 1 & 1 & 1 & 1 & 1 & 1 & 0 & 1 & 1 & 1 \\
\hline $890-901-172-4$ & Cooperativa Consumo & 1 & 0 & 0 & 0 & 0 & 0 & 0 & 0 & 0 & 0 \\
\hline 890-901-188-1 & Superfondo Fondo de Empleados & 0 & 0 & 0 & 0 & 0 & 0 & 0 & 0 & 0 & 0 \\
\hline 890-901-502-1 & Fondo de Empleados del Grupo Bancolombia & 1 & 1 & 1 & 1 & 1 & 1 & 1 & 1 & 1 & 1 \\
\hline $890-904-478-6$ & Cooperativa Colanta-Colanta & 1 & 0 & 0 & 0 & 0 & 0 & 0 & 0 & 0 & 0 \\
\hline $890-904-843-1$ & $\begin{array}{l}\text { Coopantex Cooperativa de Ahorro y Crédito- } \\
\text { Coopantex }\end{array}$ & 1 & 0 & 0 & 0 & 0 & 0 & 0 & 0 & 0 & 0 \\
\hline $890-904-902-8$ & Cooperativa de Ahorro y Crédito Pío XII & 1 & 1 & 0 & 0 & 0 & 0 & 0 & 0 & 0 & 0 \\
\hline $890-905-085-1$ & $\begin{array}{l}\text { Cooperativa Nacional de Trabajadores- } \\
\text { Coopetraban }\end{array}$ & 1 & 1 & 1 & 1 & 1 & 1 & 0 & 1 & 1 & 1 \\
\hline $890-905-206-4$ & Cooperativa de Yarumal & 1 & 0 & 0 & 0 & 0 & 0 & 0 & 0 & 0 & 0 \\
\hline $890-905-327-7$ & Forjar Cooperativa de Ahorro y Crédito & 1 & 0 & 0 & 0 & 0 & 0 & 0 & 0 & 0 & 0 \\
\hline $890-905-574-1$ & $\begin{array}{l}\text { Cooperativa Médica de Antioquia Ltda.- } \\
\text { Comedal }\end{array}$ & 1 & 0 & 0 & 0 & 0 & 0 & 0 & 0 & 0 & 0 \\
\hline $890-905-864-0$ & $\begin{array}{l}\text { Fondo de Beneficio Común de los Empleados } \\
\text { del Sena }\end{array}$ & 1 & 1 & 1 & 1 & 1 & 1 & 1 & 1 & 1 & 1 \\
\hline $890-906-852-7$ & Cooperativa de Trabajadores del Sena & 1 & 1 & 0 & 0 & 0 & o & 0 & 0 & 0 & 0 \\
\hline $890-907-638-1$ & Cooperativa de Caficultores de Andes Ltda. & 1 & 0 & 0 & 0 & 0 & 0 & 0 & 0 & 0 & 0 \\
\hline $890-908-823-2$ & $\begin{array}{l}\text { Cooperativa Multiactiva Santa Rosa de Osos } \\
\text { Ltda. }\end{array}$ & 1 & 0 & 0 & 0 & 0 & 0 & 0 & 0 & 0 & 0 \\
\hline $890-909-246-7$ & Cooperativa Belén Ahorro y Crédito-Cobelén & 1 & 0 & 0 & 0 & 0 & 0 & 0 & 0 & 0 & 0 \\
\hline $890-911-402-6$ & Cooperativa Suya & 1 & 0 & 0 & 0 & 0 & 0 & 0 & 0 & 0 & 0 \\
\hline
\end{tabular}




\begin{tabular}{|c|c|c|c|c|c|c|c|c|c|c|c|}
\hline NIT & NOMBRE DE LA ENTIDAD-SIGLA & A & B & C & D & $\mathrm{E}$ & $\mathrm{F}$ & G & H & I & $J$ \\
\hline $890-981-459-4$ & $\begin{array}{l}\text { Cooperativa de Ahorro y Crédito Crear Ltda.- } \\
\text { Crearcop }\end{array}$ & 1 & 0 & 0 & 0 & 0 & 0 & 0 & 0 & 0 & 0 \\
\hline $890-981-912-1$ & $\begin{array}{l}\text { Cooperativa San Pío x de Granada Ltda.- } \\
\text { Coogranada }\end{array}$ & 1 & 0 & 0 & 0 & 0 & 0 & 0 & 0 & 0 & 0 \\
\hline $890-982-409-0$ & Comfamigos Cooperativa Multiactiva & 1 & 0 & 0 & 0 & 0 & 0 & 0 & 0 & 0 & 0 \\
\hline 890-984-981-1 & Cooperativa de Ahorro y Crédito Servunal & 1 & 1 & 1 & 1 & 1 & 1 & 1 & 1 & 1 & 1 \\
\hline $890-985-032-1$ & $\begin{array}{l}\text { Cooperativa de Profesores de la Universidad } \\
\text { de Antioquia- Cooprudea }\end{array}$ & 1 & 0 & 0 & 0 & 0 & 0 & 0 & 0 & 0 & 0 \\
\hline $890-985-077-2$ & $\begin{array}{l}\text { Cooperativa Multiactiva Universitaria } \\
\text { Nacional }\end{array}$ & 1 & 1 & 1 & 1 & 1 & 1 & 1 & 1 & 1 & 1 \\
\hline $890-985-280-1$ & $\begin{array}{l}\text { Fondo de Empleados de Bancolombia S.A. y } \\
\text { Filiales-FEC }\end{array}$ & 1 & 0 & 0 & 0 & 0 & 0 & 0 & 0 & 0 & 0 \\
\hline $891-100-079-3$ & $\begin{array}{l}\text { Cooperativa de Ahorro y Crédito San Miguel- } \\
\text { Coofisam }\end{array}$ & 1 & 0 & 0 & 0 & 0 & 0 & 0 & 0 & 0 & 0 \\
\hline $891-100-656-3$ & $\begin{array}{l}\text { Cooperativa Nacional Educativa de Ahorro y } \\
\text { Crédito-Coonfie }\end{array}$ & 1 & 0 & 0 & 0 & 0 & 0 & 0 & 0 & 0 & 0 \\
\hline $891-100-673-9$ & $\begin{array}{l}\text { Cooperativa Latinoamericana de Ahorro y } \\
\text { Crédito-Utrahuilca }\end{array}$ & 1 & 0 & 0 & 0 & 0 & 0 & 0 & 0 & 0 & 0 \\
\hline $891-300-716-5$ & Cooperativa de Ahorro y Crédito Cootraipi & 1 & 1 & 0 & 0 & 0 & 0 & 0 & 0 & 0 & 0 \\
\hline 891-301-208-1 & Cooperativa Trabajadores Ingenio Mayaguez & 1 & 0 & 0 & 0 & 0 & 0 & 0 & 0 & 0 & 0 \\
\hline $891-400-646-7$ & $\begin{array}{l}\text { Cooperativa del Municipio de Pereira y } \\
\text { Departamento de Risaralda }\end{array}$ & 0 & 0 & 0 & 0 & 0 & 0 & 0 & 0 & 0 & \\
\hline $891-409-747-3$ & $\begin{array}{l}\text { Cooperativa de Trabajadores de la } \\
\text { Educacion del Risaralda }\end{array}$ & 1 & 0 & 0 & 0 & 0 & 0 & 0 & 0 & 0 & 0 \\
\hline $891-701-124-6$ & Cooperativa de Educadores del Magdalena & 1 & 0 & 0 & 0 & 0 & 0 & 0 & 0 & 0 & o \\
\hline $891-801-371-8$ & $\begin{array}{l}\text { Coperativa Multiactiva de Educadores de } \\
\text { Boyacá-Coeducadores Boyacá }\end{array}$ & 1 & 0 & 0 & 0 & o & 0 & 0 & 0 & 0 & 0 \\
\hline 892-000-373-9 & Cooperativa de Ahorro y Crédito Congente & 1 & 0 & 0 & 0 & 0 & 0 & 0 & 0 & 0 & 0 \\
\hline $900-062-612-8$ & $\begin{array}{l}\text { Cooperativa Emssanar Servicio } \\
\text { Farmacéutico }\end{array}$ & 1 & 1 & 0 & 0 & 0 & 0 & 0 & 0 & 0 & 0 \\
\hline $900-067-659-6$ & Cooperativa Epsifarma-Epsifarma & 0 & 0 & 0 & 0 & 0 & 0 & 0 & 0 & 0 & 0 \\
\hline $900-163-087-4$ & $\begin{array}{l}\text { Cooperativa de Ahorro y Crédito de } \\
\text { Droguistas Detallistas-Copicredito }\end{array}$ & 1 & 0 & 0 & 0 & 0 & 0 & 0 & 0 & 0 & 0 \\
\hline $900-175-962-6$ & Cooperativa de Ahorro y Crédito Colanta & 1 & 0 & 0 & 0 & 0 & 0 & 0 & 0 & 0 & 0 \\
\hline $900-189-084-5$ & $\begin{array}{l}\text { Microempresas de Colombia Cooperativa } \\
\text { de Ahorro y Crédito- Microempresas de } \\
\text { Colombia A.C. }\end{array}$ & 1 & 0 & 0 & 0 & 0 & 0 & 0 & 0 & 0 & 0 \\
\hline $900-460-059-1$ & $\begin{array}{l}\text { Coperativa Especializada de Ahorro y } \\
\text { Crédito Canapro }\end{array}$ & 1 & 1 & 0 & 0 & 0 & 0 & 0 & 0 & 0 & 0 \\
\hline
\end{tabular}

